\title{
Education and Successful Aging Trajectories: A Longitudinal Population-Based Latent Variable Modelling Analysis
}

\author{
Theodore D. Cosco, ${ }^{1,2}$ Blossom C.M. Stephan, ${ }^{4}$ Carol Brayne, ${ }^{3}$ Graciela Muniz, ${ }^{5}$ and MRC CFAS
}

\begin{abstract}
As the population ages, interest is increasing in studying aging well. However, more refined means of examining predictors of biopsychosocial conceptualizations of successful aging (SA) are required. Existing evidence of the relationship between early-life education and later-life SA is unclear. The Successful Aging Index (SAI) was mapped onto the Cognitive Function and Aging Study (CFAS), a longitudinal population-based cohort $(n=1,141)$. SAI scores were examined using growth mixture modelling (GMM) to identify SA trajectories. Unadjusted and adjusted (age, sex, occupational status) ordinal logistic regressions were conducted to examine the association between trajectory membership and education level. GMM identified a three-class model, capturing high, moderate, and low functioning trajectories. Adjusted ordinal logistic regression models indicated that individuals in higher SAI classes were significantly more likely to have higher educational attainment than individuals in the lower SAI classes. These results provide evidence of a life course link between education and SA.
\end{abstract}

\section{RÉSUMÉ}

Le vieillissement de la population a non seulement accru l'intérêt pour les études concernant les problèmes de santé, mais aussi pour celles associées au vieillissement en santé. Cependant, il serait nécessaire de définir des mesures plus raffinées liées aux prédicteurs compris dans les conceptualisations biopsychosociales du vieillissement réussi (VR). Les données probantes recueillies à ce jour concernant les liens entre l'éducation à un jeune âge et le VR ne sont pas encore claires. L'indice de vieillissement réussi (IVR) a été cartographié dans le cadre de l'étude Cognitive Function and Aging Study (CFAS), impliquant une cohorte populationnelle étudiée dans une perspective longitudinale $(n=1141)$. Les scores IVR ont été examinés selon l'approche du growth mixture modelling (GMM) afin d'identifier des trajectoires de VR. Des régressions logistiques ordinales pondérées (selon l'âge, le sexe, le statut professionnel) et non pondérées ont été réalisées pour évaluer l'association entre les types de trajectoires et le niveau d'éducation. Le GMM a permis d'identifier un modèle à trois classes, comprenant des trajectoires de fonctionnement hautes (TFH), modérées (TFM) et basses (TFB). Les régressions logistiques ordinales pondérées ont mis en évidence que les individus des classes d'IVR supérieures présentaient une plus forte probabilité d'avoir atteint un niveau de scolarité plus élevé que les individus à IVR inférieur (TFM, TFB) dans cet échantillon (RR 1.44, IC 95 \% 1.14-1.82). L'éducation à un jeune âge est associée de manière indépendante à des trajectoires de VR supérieures plus tard dans la vie. Ces résultats recueillis dans une cohorte de sujets britanniques âgés démontrent l'impact du parcours de vie par des liens entre l'éducation et le VR, et révèlent l'influence bénéfique de l'éducation dans le long terme.

1 Oxford Institute of Population Ageing, University of Oxford

2 MRC Unit for Lifelong Health and Ageing, University College London

3 Cambridge Institute of Public Health, University of Cambridge

4 Institute of Health and Society, Newcastle University

5 Centre for Clinical Brain Sciences, University of Edinburgh

Manuscript received: / manuscrit reçu : 09/03/16

Manuscript accepted: / manuscrit accepté : 25/02/17

Keywords: aging, successful aging, growth mixture modelling, healthy aging, educational status

Mots-clés : vieillissement, vieillissement réussi, growth mixture modelling, vieillissement en santé, niveau de scolarité 
Correspondence and requests for reprints should be sent to / La correspondance et les demandes de tirés à part doivent être adressées à :

Theodore D. Cosco, Ph.D.

Oxford Institute of Population Ageing

66 Banbury Road

University of Oxford

Oxford, OX2 6PR

UK

<tdcosco@cantab.net>

\section{Background}

The number of individuals aged 65 and older is expected to exceed the number of individuals aged below age 15 by 2045, worldwide (United Nations, 2010). Human life expectancy has been increased through advances in medical technology and practice, as well as through changes in social and public health. However, these additional years may not be experienced with good physical health, cognitive functioning, and / or psychosocial well-being. Fostering physiological and psychosocial well-being across the lifespan has important health, policy, and economic implications to mitigate the global demographic shift (United Nations, 2010). In addition to studying specific disorders and the negative aspects of aging, research into the ways in which individuals age particularly well can provide insights into whether and how the latter years of life might be improved.

In the absence of a consensus definition of successful aging (SA) (Cosco, Prina, Perales, Stephan, \& Brayne, 2014), researchers have called for more refined measures (Cosco, Stephan, \& Brayne, 2014; Kivimaki \& Ferrie, 2011). Kivimaki and Ferrie (2011) have identified important shortcomings both in the ability of extant SA metrics to capture SA and in the ways we examine heterogeneity in the aging process. To address these issues, the SA model needs to be grounded in real populations, to be relevant to older people themselves, and to be implemented with sufficient detail to capture the heterogeneity of aging, which includes going beyond biomedical conceptualizations of SA (Bowling \& Iliffe, 2006). In addition to improvement in capturing SA, methods of analysis of such measures in populations over time need to be used. Growth mixture modelling (GMM) is a person-centred, longitudinal latent-variable modelling technique used to identify heterogeneous classes of individuals' responses on a continuous (or ordinal categorical) variable (Muthen et al., 2002), permitting further analysis of the relationship between variables and class membership. GMM has been previously used in the context of disease states, but it has rarely been attempted in the exploration of positive states of aging. In contrast to studies examining individuals' ill-health, the current study examined the unique characteristics of individuals in the highest functioning longitudinal trajectories.

Several studies to date have examined the relationship between SA and education (Liang et al., 2003; Montross et al., 2006; Palmore, 1979; Strawbridge, Cohen, Shema, \& Kaplan, 1996), with mixed results. Using exclusively biomedical SA models, Strawbridge et al. (1996) and Ford et al. (2000) failed to demonstrate a relationship between education and SA. Further, an investigation into self-rated SA and education revealed no significant relationship (Montross et al., 2006). In contrast to these one-dimensional models, Palmore (1979), Vaillant and Mukamal (2001), and FernandezBallesteros Garcia et al. (2011) employed multidimensional models of SA. Palmore identified no significant relationship, Fernandez-Ballesteros Garcia identified one significant relationship among four unique models, and Vaillant found that for each additional year of education the likelihood of an individual's having poor physical and psychosocial well-being was reduced by 0.85 (95\% CI [0.77-0.96]). These studies highlight differences in mapping of SA and the conflicting results between different models of SA and education.

In the current study we used a multidimensional model of SA, developed a priori, to examine the association between education and longitudinal trajectories of SA in later life in a population-representative cohort of adults aged 65 years and older.

\section{Methods}

\section{Study Characteristics}

The Cognitive Function and Aging Study (CFAS) is a population-based, multicentre cohort study of community-dwelling individuals $(n=13,004)$ aged 65 years and older. Baseline interviewing began in 1991 in five centres using identical methodology in England and Wales (Newcastle, Nottingham, Oxford, Cambridgeshire, and Gwynedd) (Brayne, 2006). A 20 per cent $(n=2,640)$ stratified sample (selected based on cognitive ability, age, and centre) completed a more detailed assessment 
interview, with re-interviewing approximately every two years. Data from over four years' follow-up were used in this analysis.

Trained interviewers conducted face-to-face interviews in participants' place of residence. Questions concerning demographics, cognition (Mini-Mental State Exam: MMSE; Folstein, Robins, \& Helzer, 1983), activities of daily living (ADLs), instrumental activities of daily living (IADLs) (Townsend \& Ryan, 1991), and psychosocial well-being were included in the interview. Further details of the sampling methods and interview questionnaires are available at www.cfas. ac.uk (Brayne, 2006).

In keeping with the availability of relevant variables and missingness (as outlined in the next section), in this analysis we used data from participants who had completed all of the required components of the successful aging index (SAI) at the second wave of data collection and the two subsequent waves of data collection (data version 9.0) $(n=1,141)$. All study centres obtained ethical approval from local research ethical committees.

\section{Successful Aging}

The Successful Aging Index (SAI) is a validated biopsychosocial measure of healthy aging (Cosco, Stephan, \& Brayne, 2015), created using components identified by systematic reviews of lay perspectives that is, qualitative interviews with members of the general public (Cosco, Prina, Perales, Stephan, \& Brayne, 2013) and researchers' operational definitions (Cosco, Prina, et al., 2014) of SA. The SAI includes measures of cognition (Mini-Mental State Examination), physical functioning (Activities of Daily Living [Townsend \& Ryan, 1991]; Instrumental Activities of Daily Living [Lawton \& Brody, 1969]), personal resources (e.g., optimism), self-awareness (e.g., selfrated health), and engagement (e.g., interest). The SAI score ranges from 0 to 100 with higher scores indicating greater levels of biopsychosocial SA.

\section{Education}

Education was captured via a single question asking individuals how many years they had spent in fulltime education. Participants were grouped into 0-9, $10-11$, and $\geq 12$ years of full-time education reflecting basic, moderate, and high educational attainment in this generation (Collerton et al., 2007).

\section{Co-variates}

Occupational status was defined using an individual's occupation at baseline, divided into manual and nonmanual occupations. Marital status was grouped into married/cohabiting or not married, including separated, widowed, or single.

\section{Statistical Procedures \\ Growth Mixture Modelling}

We modelled scores from each participant's SAI by using GMM to identify groups of individuals with similar SA trajectories, adjusting class-specific trajectory parameters by sex and age at first occasion. Using GMM procedures, we plotted SAI scores from three waves of CFAS data collection (each two years apart) and identified heterogeneous trajectories of SA. Models were estimated using maximum likelihood estimation, with robust estimates under a missing-at-random assumption. Given that only three waves of data were collected, we did not test non-linear trajectories as they require four or more waves of data (Bollen \& Curran, 2006). All GMM procedures were conducted in MPlus v7.1 (Muthen \& Muthen, 1998-2011).

Recommended model selection procedures involve the examination of fit indices - for example, Bayesian Information Criterion (BIC) (Raftery, 1995), interpretation of classes, and classification properties. As the number of classes is not known a priori, we fitted models with an increasing number of classes and chose the model with the lowest BIC (Schwarz, 1978). In addition to the standard BIC, the sample-adjusted BIC (SABIC) (Sclove, 1987) and Akaike Information Criterion (AIC) (Akaike, 1973) were assessed to provide supporting evidence of the fit of the model, with lower SABIC and AIC scores indicating better model fit. Models that include particularly small portions (e.g., $<1 \%$ ) of the sample have limited practical applicability, a criterion that is also used to evaluate model fit.

The model requires a stage of interpretation of classes since spurious classes may be identified (Bauer \& Curran, 2003) resulting from the nature of the model, which has been designed to fit non-normal data. Classification is assessed via evaluation of the entropy, an index that takes values between 0 and 1 with high values indicating a clear classification of individuals in classes (Celeux \& Soromenho, 1996).

\section{Analysis of Education and SAI Trajectory}

Once we had identified the best fitting model, we performed an a posteriori analysis of the data to examine differences in education group and trajectory membership. We used chi-square and $t$-tests to examine group differences in demographic variables including age, marital status (married or not married), occupational status (manual employment or non-manual employment), and sex (female or male). We examined the differences in educational level between individuals in 
the highest SA trajectory and the lower SA groups in the total sample and by gender using the OLOGIT command in Stata 14. To accomplish this, we used unadjusted and adjusted (age, sex, marital status, occupational status) ordinal logistic regressions (backweighted to adjust for over sampling of individuals aged 75 years or older and sampling to the diagnostic interview at baseline). We further examined interaction/ effect modification by conducting age, sex, and occupational status and multicollinearity via variance inflation factor analysis (with values greater than 10 identified as problematic).

\section{Missingness}

Given that the SAI is an average of all the constituent components, we excluded individuals with missing component values and conducted a complete case analysis. This was done as missing data on one or more component variables would skew calculation of the SAI variable, providing an unrepresentative score for those individuals without complete data. Missingness at random for demographic variables in the multivariate modelling was assessed using a chi-square test examining the association between missingness and years in full-time education.

\section{Results}

\section{Sample Characteristics}

The sample included 1,141 individuals, with a mean age at baseline of 76.39 (standard deviation $[S D]=6.47$ ). The sample was primarily female $(63.37 \%)$, not married $(51.89 \%)$, had manual occupations $(70.20 \%)$ and $0-9$ years of fulltime education $(64.25 \%)$ (Table 1$)$. At the first follow-up wave, 619 participants (54.3\%) remained in the study, and at the second follow-up, $144(12.4 \%)$ remained. Missingness was significantly associated with sex (greater missingness in women: $n=360[33.24 \%]$, compared to men: $n=151[26.54 \%]$; $\chi^{2}=7.84, p=.005$ ), older age (mean age for missing participants 83.05; mean age for participants in sample $76.39 ; t[1,650]=-17.99, p<.001$ ), marital status (missing if married: 143 [20.61\%]; missing if not married, 284 $[32.57 \%] ; \chi^{2}=35.76, p<.001$ ) and education (missing if 0-9 years education: 295 [28.89\%]); missing if 10-11 years education: 76 [23.24\%]; missing if $\geq 12$ years education: $\left.36[19.05 \%] ; \chi^{2}=10.18, p=.006\right)$. No significant differences were identified between missingness and occupational status (missing if in manual occupation: 333 [29.68\%]; missing if in non-manual position: 173 $\left.[34.06 \%] ; \chi^{2}=3.13, p=.08\right)$.

\section{Trajectory Analysis}

The GMM procedure revealed three distinct SA classes: the highest functioning trajectory (HFT; $n=125$ ), moderate functioning trajectory (MFT; $n=458$ ), and low functioning trajectory (LFT; $n=558$ ). The HFT class had the highest intercept and flattest slope; the MFT had a moderate intercept and slope; and the LFT had the lowest intercept and steepest slope (Figure 1). The threeclass model was selected according to the AIC and BIC fit indices in combination with the entropy and theoretical relevance of these findings (Table 2); this model presented the greatest entropy (0.66) in combination with the lowest BIC (1525.880). Although the four-class model presented a lower AIC and SABIC, the inability of the model to converge and the much lower entropy and higher BIC suggested that this model was a poorer fit when compared to the three-class model.

\section{Demographic Characteristics}

When compared to the MFT and LFT classes, individuals in the HFT class were significantly younger,

Table 1: Baseline sample demographic characteristics

\begin{tabular}{|c|c|c|c|c|c|c|c|c|c|c|c|c|}
\hline & \multirow[b]{2}{*}{$n$} & \multicolumn{3}{|c|}{ Age } & \multirow[b]{2}{*}{ Women (\%) } & \multirow[b]{2}{*}{ Married (\%) } & \multirow{2}{*}{$\begin{array}{c}\text { Low Occupational } \\
\text { Status(\%) }\end{array}$} & \multicolumn{3}{|c|}{ Education (\%) } & \multicolumn{2}{|c|}{$\begin{array}{l}\text { Trajectory } \\
\text { (SAI Score) }\end{array}$} \\
\hline & & Mean & $S D$ & Range & & & & $0-9$ & 10,11 & $12+$ & Intercept & Slope \\
\hline $\begin{array}{l}\text { Total Sample } \\
\text { Classes }\end{array}$ & 1141 & 76.39 & 6.47 & $66-100$ & 63.37 & 48.11 & 70.20 & 64.25 & 22.21 & 13.54 & & \\
\hline $\mathrm{HFT}^{\mathrm{a}}$ & 125 & 72.64 & 4.77 & $67-90$ & 35.20 & 78.40 & 48.04 & 45.28 & 27.36 & 27.36 & 91.98 & -0.29 \\
\hline \multicolumn{13}{|l|}{ Years Education } \\
\hline $0-9 b$ & 726 & 76.22 & 6.49 & $66-95$ & 61.29 & 47.11 & 80.25 & & & & & \\
\hline 10,11 & 251 & 76.65 & 6.52 & $66-100$ & 63.75 & 51.39 & $64.34^{* * *}$ & & & & & \\
\hline $12+$ & 153 & 76.46 & 6.27 & $67-93$ & 71.90 & 50.98 & $32.67^{* * *}$ & & & & & \\
\hline
\end{tabular}

a,b Comparison group; ${ }^{* *} p<.01 ;^{* * *} p<.001$; Low occupational status: manual employment

HFT = high functioning trajectories; LFT = low functioning trajectories; MFT = moderate functioning trajectories 


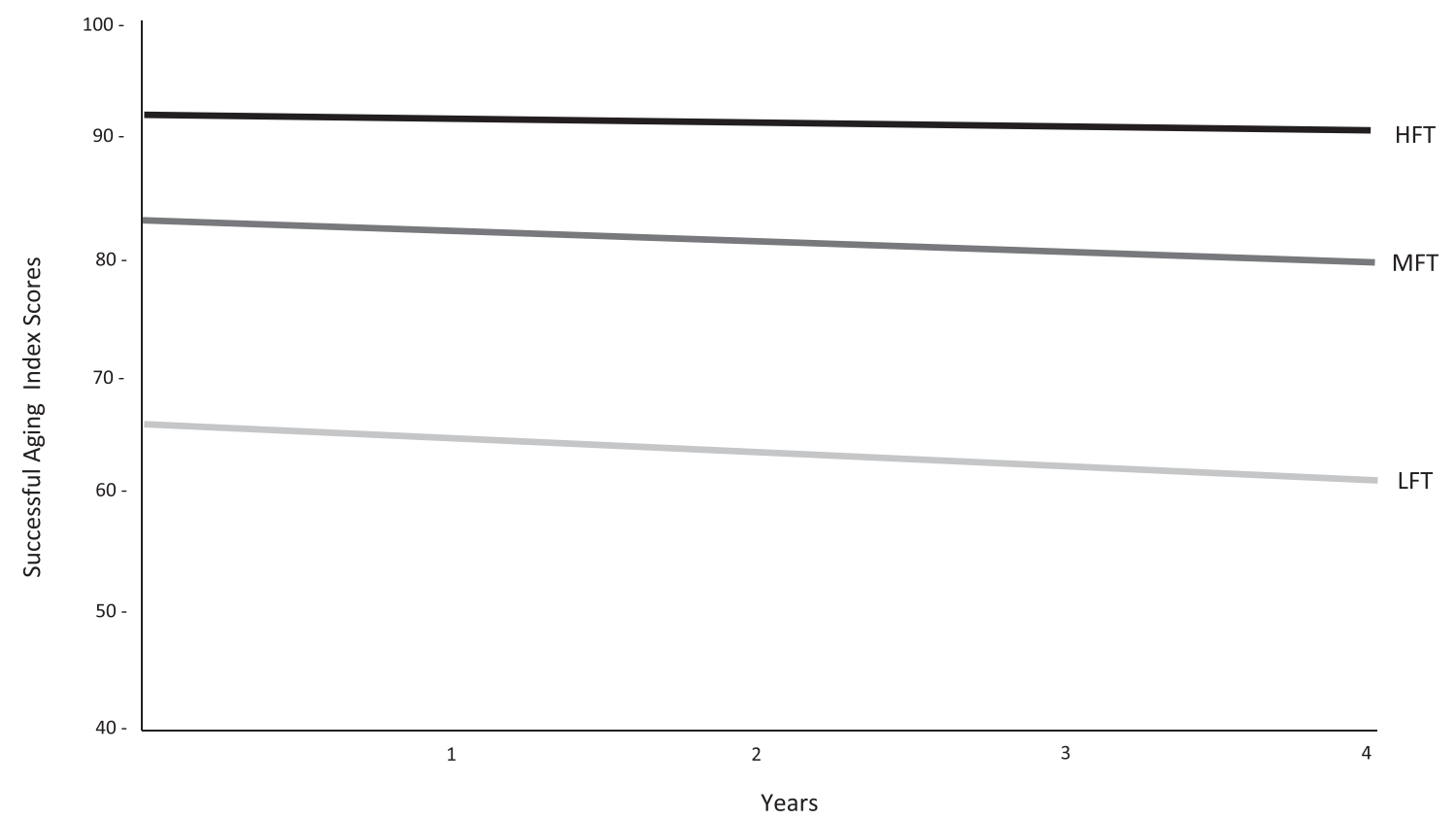

Figure 1: Estimated mean trajectories of Successful Aging Index scores (HFT: high functioning trajectories; MFT: moderate functioning trajectories; LFT: low functioning trajectories).

composed of more men, had higher occupational status, and were more likely to be married (Table 1).

\section{Education and Successful Aging}

In the total sample, individuals with more education were significantly more likely to be in higher functioning classes in unadjusted (OR 1.38, 95\% CI [1.13-1.69]) and adjusted models (age, occupational status, marital status, sex) (OR 1.44, 95\% CI [1.14-1.82]). Among men, a relationship between education and successful aging class existed in an unadjusted (OR 1.54, 95\% CI [1.09-2.18]) but not in an adjusted model (OR 1.31, 95\% CI [0.90-1.92]). Among women, a relationship between education and successful aging class existed in unadjusted (OR 1.60, 95\% CI [1.24-2.07]) and adjusted models (OR 1.50, 95\% CI [1.11-2.03]). Men were significantly more likely to be in higher functioning classes if they had more education in unadjusted models; however, this relationship was attenuated by age, sex, and marital status in adjusted models.

Table 2: Model selection criteria

\begin{tabular}{lccccc}
\hline Class & AIC & BIC & SABIC & Entropy & (\% of sample) \\
\hline 2 & 14491.776 & 14557.291 & 14515.999 & 0.57 & 41.5 \\
3 & 14420.047 & 14525.880 & 14459.178 & 0.66 & 10.4 \\
$4^{*}$ & 14400.269 & 14556.499 & 14458.034 & 0.59 & 10.6 \\
\hline
\end{tabular}

Note: *Did not converge; AIC: Akaike Information Criteria; BIC: Bayesian Information Criteria; SABIC: Sample-adjusted Bayesian Information Criteria.
Women were significantly more likely to be in higher functioning classes in unadjusted and adjusted (age, occupational status, marital status) models if they had higher levels of educational attainment. The relationship between education and functional class membership was, however, attenuated by 14.9 per cent when controlling for age, occupational status, and marital status. In the full sample, fully adjusted models indicated that higher education was associated with higher functioning classes.

No statistically significant interactions were observed between education and sex (men as reference group; 10-11 years' education: OR $0.86,95 \%$ CI $[0.48,1.55]$; $\geq 12$ years' education: OR $1.37,95 \%$ CI $[0.65,2.88])$, age (10-11 years' education: OR 1.19, 95\% CI [0.67, 2.11]; $\geq 12$ years' education: OR $1.00,95 \%$ CI $[0.50,1.99])$, occupational status (manual occupation as reference group; 10-11 years' education: OR 1.21, 95\% CI [0.65, 2.25]; $\geq 12$ years' education: OR $0.68,95 \%$ CI $[0.32,1.44])$, or marital status (married participants as reference group; 10-11 years' education: OR 1.01, 95\% CI [0.57, 1.77]; $\geq 12$ years' education: OR $1.74,95 \%$ CI $[0.88,3.44])$. Further, variance inflation factor (VIF) analysis revealed no evidence of multicollinearity with respect to education $(\mathrm{VIF}=1.15)$, age $(\mathrm{VIF}=1.11)$, sex $(\mathrm{VIF}=1.09)$, occupational status (VIF $=1.15)$, or marital status $(\mathrm{VIF}=1.18)$.

\section{Discussion}

Using GMM procedures, we identified high, moderate, and low SA trajectory classes. Individuals in the HFT 
class were primarily men, married, and of high occupational status. After adjusting for age, sex, and occupational status, a significant and independent association between higher education and better SA trajectories in later life was demonstrated in the total sample. These results suggest that early-life education is an independent marker of SA in later life.

Limitations include a substantial degree of missing data and model fit. As highlighted in the methods, our study used a complete case analysis. We made this decision to ensure that the integrity of the SAI was maintained, that is, that respondents' subjective interpretation of components was captured. Further, as in any longitudinal study of older adults, there is a significant level of attrition via death. This attrition predisposes the sample to having a survivor bias in which only individuals who are healthier are included (Young, Powers, \& Bell, 2006). Given the association between longevity and indicators of socioeconomic advantage, such as education, this may also result in more educated individuals staying in the sample (Young et al., 2006), as was the case in the current study. Although individuals who were less healthy and less educated may not have been included in the sample, one purpose of this study was to examine the association between the educational attainment of individuals in the best functioning successful aging trajectory relative to other individuals in the sample, even if they were part of a particularly healthy and well-educated group. As a result of this attrition, subsequent waves of the CFAS (e.g., beyond four years' follow-up) could not be used because of the inability of the models to converge. However, GMM uses maximum likelihood estimation, with robust estimates under a missingness-at-random assumption.

Missingness in the dataset was assessed with respect to age, sex, education, and marital status; we noted that individuals missing from the current study but included in the broader CFAS were significantly older and had significantly fewer years of full-time education. An additional purpose of the current study was to identify individuals who were aging particularly well within the sample rather than to present trajectories that are representative of the general population. It is important to note that only a relatively small proportion of individuals in such a large study met the criteria for the high functioning HA trajectory. Further, given the observational nature of the study, we were unable to establish the underpinning causative mechanisms that were driving the relationships observed.

The best-fitting model was selected for further analysis; however, limitations in the model fit must be acknowledged, notably regarding entropy. Entropy refers to the degree to which a model can delineate between classes, with lower levels of entropy indicating a higher probability of misclassification of individuals into classes. The model we chose for further analysis had the highest level of entropy of all the possible model permutations; however, by absolute standards, rather than relative standards, this level of entropy was relatively low. Therefore, we have used the best fitting model in these analyses given the possibility of misclassification of individuals based on the entropy of the model.

Previous studies that have examined the relationship between SA and education have used mixed models and have subsequently produced contradictory results (Liang et al., 2003; Montross et al., 2006; Palmore, 1979; Strawbridge et al., 1996). Studies that employed unidimensional models (for example, only physical functioning) did not demonstrate a significant relationship between education and SA (Ford et al., 2000; Strawbridge et al., 1996). However, in three of six studies that invoked a multidimensional model of SA, including both psychosocial and biomedical components, researchers observed significant relationships (Fernandez-Ballesteros Garcia et al., 2011; Hamid, Momtaz, \& Ibrahim, 2012; Vaillant \& Mukamal, 2001). Of note, in the Fernandez-Ballesteros Garcia et al. (2011) models, the only model that reached significance was the one with the greatest number of psychosocial components, including subjective health and satisfaction. These studies used models that were primarily researcher-driven in their constituent components and in their thresholds.

Our current study used an a priori model of SA and a data-driven method for extraction of SA trajectories. The components included in the SAI have been informed by lay perspectives, given the SAI relevance to older people. A key strength of GMM models is the ability to articulate SA in relative, rather than absolute, terms. In models of SA that posit researcher-driven thresholds if individuals cannot fulfill these criteria, the opportunity for further analysis is inhibited. Conversely, in GMM, individuals' performance is groupedbased using similar trajectories - that is, GMM models do not employ an absolute threshold; therefore, these data are able to articulate heterogeneous trends.

Occupational status and education are closely linked, as demonstrated in the current study by the attenuation of the relationship between SA and education when adjusted for occupational status. In the total sample, however, education was a statistically significant, independent marker of membership in higher SA classes after adjusting for age, sex, and occupational status in the full sample and in a subsample of women. This relationship approached significance in men, but was not observed. Given the much lower sample size of 
men $(n=418)$, this may have been a function of the attenuation of statistical power. Although there were sex differences in the relationships between SA classes and education, these interactions did not reach statistical significance. These results provide support for the independent influence of education on SA. However, the practical implications of these results must be interpreted with caution. Although we demonstrated a statistically significant association, the application of these findings in real-world settings will require further research into the causative mechanisms that underpin this association. Another area for further research is into the relationship between occupational status and gender in aging populations. Although research into the implications of increased gender equity have suggested trends towards more positive women's health outcomes (Moss, 2002), this relationship has not been explored across cohorts or in the context of aging trajectories.

These results suggest that education is a statistically significant, independent marker of SA. Although the mechanisms underpinning this association require further investigation - as educational attainment will be a reflection of innate ability and childhood circumstances - the potential modifiability of educational attainment may permit societies to influence the future trajectory for their populations through policies implemented in early life. These findings are consistent with research looking at the negative elements of aging, notably dementia (Stern et al., 1994) and terminal decline (Batterham, Mackinnon, \& Christensen, 2011), highlighting the long-term benefits of higher education attainment in older samples.

\section{References}

Akaike, H. (1973). Maximum likelihood identification of Gaussian autoregressive moving average models. Biometrika, 60(2), 255-265.

Batterham, P. J., Mackinnon, A. J., \& Christensen, H. (2011). The effect of education on the onset and rate of terminal decline. Psychology and Aging, 26(2), 339-350.

Bauer, D., \& Curran, P. (2003). Distributional assumptions of growth mixture models: Implications for overextraction of latent trajectory classes. Psychological Methods, 8(3), 338-363.

Bollen, K., \& Curran, P. (2006). Latent curve models: A structural equation perspective. Hoboken, NJ: Wiley-Interscience.

Bowling, A., \& Iliffe, S. (2006). Which model of successful ageing should be used? Baseline findings from a British longitudinal survey of ageing. Age and Ageing, 35(6), 607-614.

Brayne, C. (2006). Incidence of dementia in England and Wales: The MRC cognitive function and ageing study.
Alzheimer Disease and Associated Disorders, 20(Suppl. 2), S47-S51.

Celeux, G., \& Soromenho, G. (1996). An entropy criterion for assessing the number of clusters in a mixture model. Journal of Classification, 13, 195-212.

Collerton, J., Barrass, K., Bond, J., Eccles, M., Jagger, C., James, O., \& Kirkwood, T. (2007). The Newcastle 85+ study: Biological, clinical and psychosocial factors associated with healthy ageing: Study protocol. BMC Geriatrics, $7(1), 1-14$.

Cosco, T. D., Prina, A. M., Perales, J., Stephan, B., \& Brayne, C. (2013). Lay perspectives of successful ageing: A systematic review and meta-ethnography. BMJ Open, 3(6), e002710.

Cosco, T. D., Prina, A. M., Perales, J., Stephan, B., \& Brayne, C. (2014). Operational definitions of successful aging: A systematic review. International Psychogeriatrics 26(3), 373-381.

Cosco, T. D., Stephan, B., \& Brayne, C. (2014). (Unsuccessful) binary modeling of successful aging in the oldest-old adults: A call for continuum-based measures. Journal of the American Geriatrics Society, 62(8), 1597-1598.

Cosco, T. D., Stephan, B. C., \& Brayne, C. (2015). Validation of an a priori, index model of successful aging in a population-based cohort study: The successful aging index. International Psychogeriatrics, 27(12), 1971-1977.

Fernandez-Ballesteros Garcia, R., Zamarron Cassinello, M., Lopez Bravo, M., Molina Martinez, M., Diez Nicolas, J., Montero Lopez, P., \& Schettini del Moral, R. (2011). Successful ageing: Criteria and predictors. Psychology in Spain, 15(1), 94-101.

Folstein, M., Robins, L., \& Helzer, J. (1983). The MiniMental State Examination. Archives of General Psychiatry, 40(7), 812.

Ford, A., Haug, M., Stange, K., Gaines, A., Noelker, L., \& Jones, P. (2000). Sustained personal autonomy: A measure of successful aging. Journal of Aging $\mathcal{E}$ Health, 12(4), 470-489.

Hamid, T., Momtaz, Y., \& Ibrahim, R. (2012). Predictors and prevalence of successful aging among older Malaysians. Gerontology, 58(4), 366-370.

Kivimaki, M., \& Ferrie, J. E. (2011). Epidemiology of healthy ageing and the idea of more refined outcome measures. International Journal of Epidemiology, 40(4), 845-847.

Lawton, M., \& Brody, E. (1969). Assessment of older people: Self-maintaining and instrumental activities of daily living. The Gerontologist, 9(3), 179-186.

Liang, J., Shaw, B., Krause, N., Bennett, J., Blaum, C., Kobayashi, E., \& Sugisawa, H. (2003). Changes in functional status among older adults in Japan: Successful and usual aging. Psychology \& Aging, 18(4), 684-695. 
Montross, L., Depp, C., Daly, J., Reichstadt, J., Golshan, S., Moore, D. ... Jeste, D. (2006). Correlates of self-rated successful aging among community-dwelling older adults. American Journal of Geriatric Psychiatry, 14(1), 43-51.

Moss, N. E. (2002). Gender equity and socioeconomic inequality: A framework for the patterning of women's health. Social Science \& Medicine, 54(5), 649-661.

Muthen, B., Brown, C., Masyn, K., Jo, B., Khoo, S., Yang, C. ... Liao, J. (2002). General growth mixture modeling for randomized preventive interventions. Biostatistics, 3(4), 459-475.

Muthen, L., \& Muthen, B. (1998-2011). Mplus User's Guide (6th ed.). Los Angeles, CA: Author.

Palmore, E. (1979). Predictors of successful aging. Gerontologist, 19(5 Pt 1), 427-431.

Raftery, A. (1995). Bayesian model selection in social research. Sociological Methodology, 25, 111-163.

Schwarz, G. (1978). Estimating dimension of a model. Annals of Statistics, 6(2), 461-464.
Sclove, S. (1987). Application of model-selection criteria to some problems in multivariate-analysis. Psychometrika, 52(3), 333-343.

Stern, Y., Gurland, B., Tatemichi, T. K., Tang, M. X., Wilder, D., \& Mayeux, R. (1994). Influence of education and occupation on the incidence of Alzheimer's disease. JAMA, 271(13), 1004-1010.

Strawbridge, W., Cohen, R., Shema, S., \& Kaplan, G. (1996). Successful aging: Predictors and associated activities. American Journal of Epidemiology, 144(2), 135-141.

Townsend, E., \& Ryan, B. (1991). Assessing independence in community living. Canadian Journal of Public Health 82(1), 52-57.

United Nations. (2010). World population ageing: 1950-2050. New York, NY: Author.

Vaillant, G., \& Mukamal, K. (2001). Successful aging. The American Journal of Psychiatry, 158(6), 839-847.

Young, A. F., Powers, J. R., \& Bell, S. L. (2006). Attrition in longitudinal studies: Who do you lose? Australian and New Zealand Journal of Public Health, 30(4), 353-361. 